\title{
A reliable method for intracranial electrode implantation and chronic electrical stimulation in the mouse brain
}

Melanie Jeffrey ${ }^{1,2,4+}$, Min Lang ${ }^{1 \dagger}$, Jonathan Gane ${ }^{1}$, Chiping $\mathrm{Wu}^{1,4}$, W Mclntyre Burnham ${ }^{2,4}$ and Liang Zhang ${ }^{1,3,4^{*}}$

\begin{abstract}
Background: Electrical stimulation of brain structures has been widely used in rodent models for kindling or modeling deep brain stimulation used clinically. This requires surgical implantation of intracranial electrodes and subsequent chronic stimulation in individual animals for several weeks. Anchoring screws and dental acrylic have long been used to secure implanted intracranial electrodes in rats. However, such an approach is limited when carried out in mouse models as the thin mouse skull may not be strong enough to accommodate the anchoring screws. We describe here a screw-free, glue-based method for implanting bipolar stimulating electrodes in the mouse brain and validate this method in a mouse model of hippocampal electrical kindling.
\end{abstract}

Methods: Male C57 black mice (initial ages of 6-8 months) were used in the present experiments. Bipolar electrodes were implanted bilaterally in the hippocampal CA3 area for electrical stimulation and electroencephalographic recordings. The electrodes were secured onto the skull via glue and dental acrylic but without anchoring screws. A daily stimulation protocol was used to induce electrographic discharges and motor seizures. The locations of implanted electrodes were verified by hippocampal electrographic activities and later histological assessments.

Results: Using the glue-based implantation method, we implanted bilateral bipolar electrodes in 25 mice. Electrographic discharges and motor seizures were successfully induced via hippocampal electrical kindling. Importantly, no animal encountered infection in the implanted area or a loss of implanted electrodes after 4-6 months of repetitive stimulation/recording.

Conclusion: We suggest that the glue-based, screw-free method is reliable for chronic brain stimulation and high-quality electroencephalographic recordings in mice. The technical aspects described this study may help future studies in mouse models.

Keywords: Glue, Kindling, Mice

\section{Background}

Electrical kindling of limbic structures in rodents is a widely used model for complex partial seizures with secondary generalization [1-4]. This model requires implantation of intracranial electrodes and subsequent brain stimulation for several weeks. The majority of

\footnotetext{
* Correspondence: liangz@uhnres.utoronto.ca

${ }^{\dagger}$ Equal contributors

'Toronto Western Research Institute, University Health Network, Toronto, Ontario, Canada

${ }^{3}$ Department of Medicine (Division of Neurology), University of Toronto,

Toronto, Ontario, Canada

Full list of author information is available at the end of the article
}

previous studies have employed this model in rats, using anchoring screws and dental acrylic to secure the implanted electrodes [5-9]. Recent studies have increasingly applied the kindling model in mice largely due to the availability of transgenic mouse strains [10-13].

In general, it is a technical challenge to firmly mount intracranial electrodes in mice with the anchoring screw/dental acrylic approach, since the mouse's thin skull may not be strong enough to accommodate the anchoring screws. As an alternative approach, we have previously developed a screw-free, glue-based method for implanting intracranial electrodes in mice [14]. Using

\section{Biomed Central}


this method, we have carried out chronic intracranial electroencephalographic (EEG) recordings in several mouse models [15-20]. In these studies, a pre-assembled electrode array was used. While the glue-based method can be used for implanting bipolar stimulating electrodes in the mouse brain [21], its validity for the chronic brain stimulation in mice that is required for kindling has not been vigorously assessed. The aim of our present study is to address this issue. We thus further modified the glue-based implantation method and conducted hippocampal electrical kindling in adult mice. EEG discharges and behavioral seizures were consistently observed in kindled mice. Importantly, no animal encountered infection in the implanted area or a loss of implanted electrodes after 4-6 months of repetitive stimulation/ recording. We thus suggest that the glue-based method is reliable for chronic brain stimulation and high-quality EEG recording in mouse models.

\section{Results}

Long-term stability of implanted electrodes and animal behavioral outcomes

25 adult male mice (initial ages of 6-8 month-old) underwent implantation of bipolar electrodes in bilateral hippocampal CA3 (Cornu Ammonis area 3). These electrodes were secured onto the skull with a modified version of the screw-free and glue-based method [14,21]. Electrode construction, assembly and mounting are schematically illustrated in Figure 1A. The surgical procedure for electrode implantation is detailed in the
A
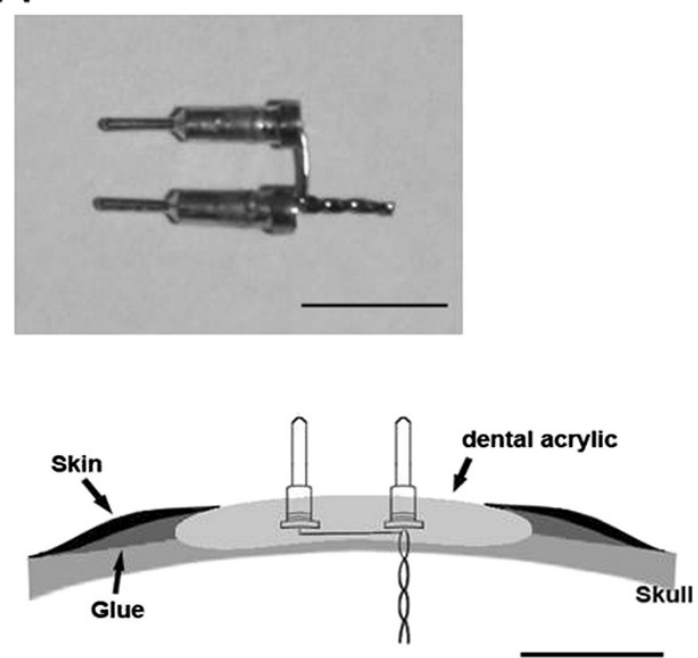

C

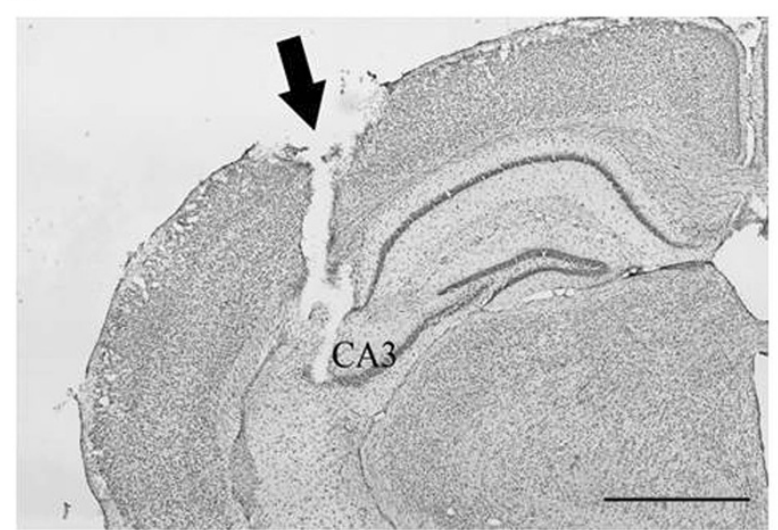

B

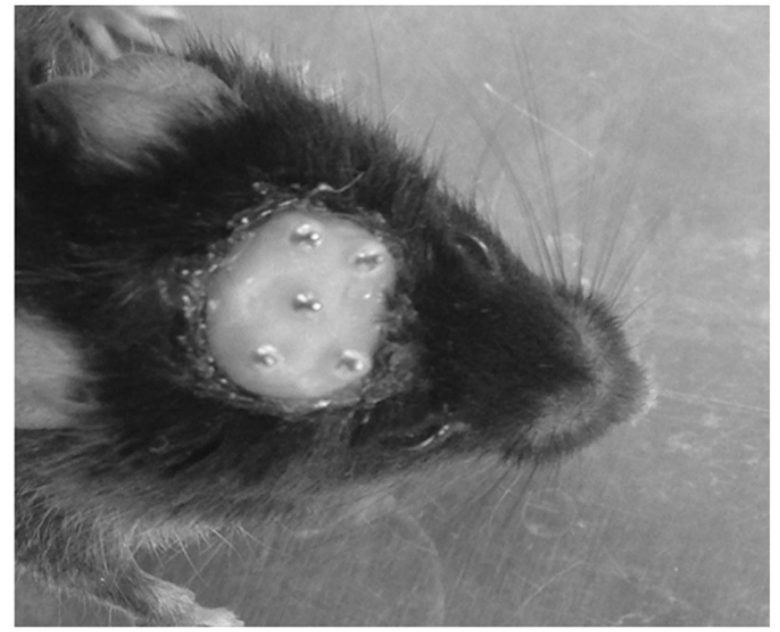

D

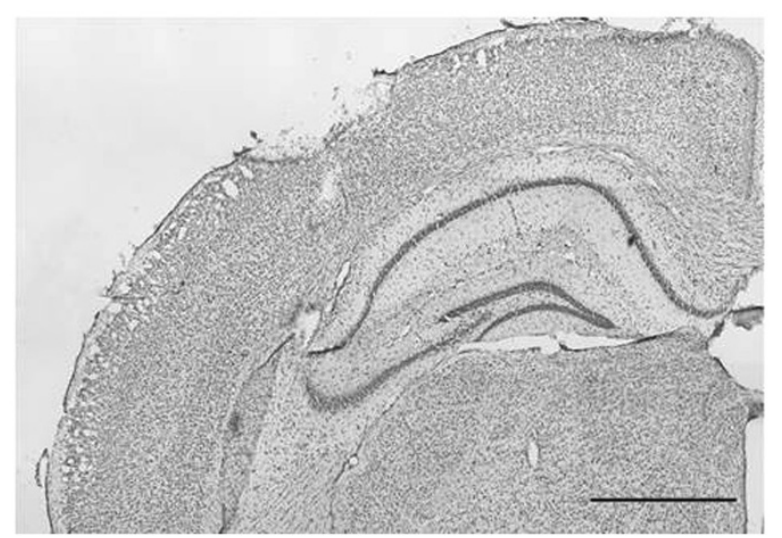

Figure 1 Illustration of connecting pins and mounted electrodes. A, a schematic illustration of electrode mounting and an image of connecting pins and a bipolar electrode. B, a photograph of a subject implanted. C-D, images of adjacent brain sections collected from a mouse. The animal was euthanized 3 months after electrode implantation. Note the track of an implanted CA3 electrode (arrowed, C) and densely packed hippocampal neurons in the area near the implanted electrode and in the adjacent section (D). Calibrations: $5 \mathrm{~mm}$ in $\mathbf{A}$ and $1 \mathrm{~mm}$ in C-D. 
Methods. Of the 25 animals implanted, we observed no evident behavioral abnormalities or spontaneous seizures in the absence of kindling stimulations. Importantly, no animal encountered local infection in the implanted area or a loss of implanted electrodes after 4-6 months of repetitive stimulation and recordings.

The locations of implanted electrodes were initially verified by in vivo electrographic activities and later, histological assessments. In response to a single stimulation of the unilateral CA3 area, synaptic field potentials were reliably recorded from the contralateral CA3 area (Figure 2A). The amplitudes of these field potentials increased with strong stimuli and reached a near plateau level at the stimulation intensity of $\geq 100 \mu \mathrm{A}$ (Figure 2B). No animals showed epileptic responses (such as long-lasting multi-spike waveforms) following a single stimulation. In
A

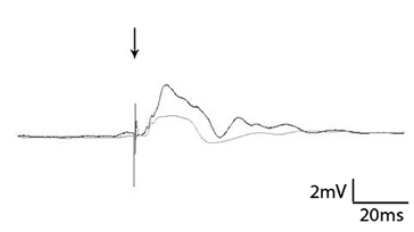

C Pre-kindling

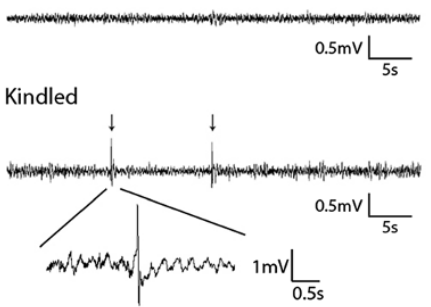

E

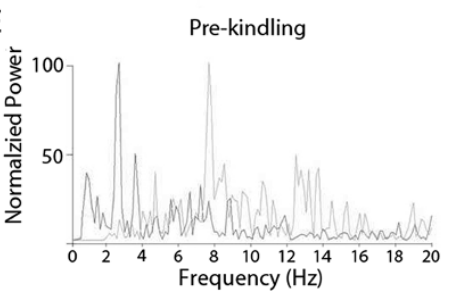

G

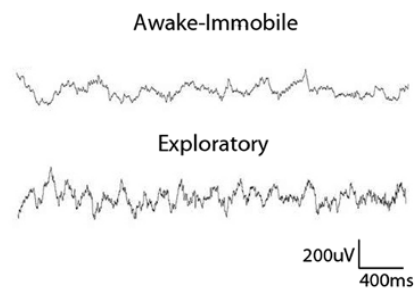

$\mathrm{B}$

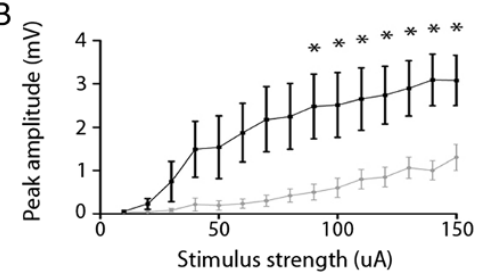

D

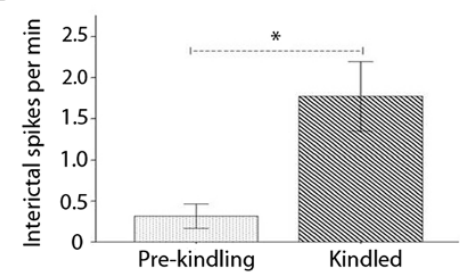

$\mathrm{F}$

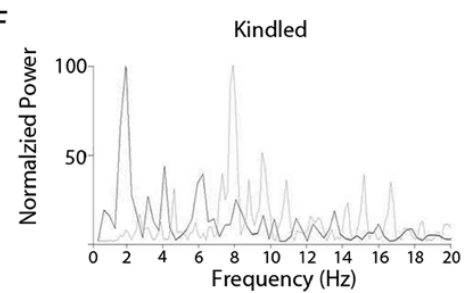

$\mathrm{H}$

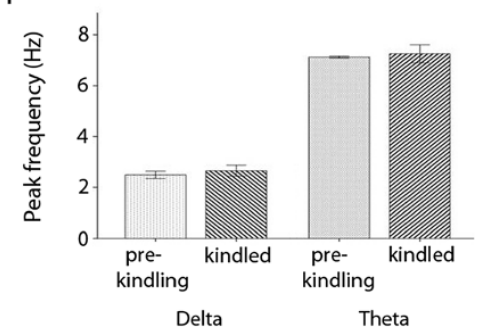

Figure 2 Electrophysiological verifications of implanted electrodes. A, representative field potentials collected from a mouse at the beginning the kindling procedure (gray) and after reaching a fully kindled state (black). These field potentials were evoked by unilateral hippocampal CA3 stimulation (arrowed) and recorded from the contralateral CA3 area. Each illustrated trace was averaged from 5 consecutive responses. The artifact of the stimulation is indicated (arrow). B, the amplitudes of CA3 field potentials measured from 5 animals pre-kindling (gray) and after kindled (black). *, initial vs. kindled responses; $\mathrm{P}<0.05$, paired t test. C, interictal-like hippocampal spikes (arrowed) recorded from a kindled animal. $\mathbf{D}$, incidences of hippocampal spikes measured pre-kindling and after fully kindled. *, $p=0.013$, paired t-test, $n=7$. E-F, representative spectral plots were generated from EEG signals that were recorded from an animal (G) pre-kindling (E) and after fully kindled (F). The spectral plots were generated from EEG segments of 30-second or 5-second during wake immobile (black) or exploratory (gray) behavior. Power was normalized to the peak of the delta band $(0.5-4 \mathrm{~Hz})$. G, representative EEG traces collected from the same animal after fully kindled. $\mathbf{H}$, peak frequencies of hippocampal theta rhythms $(5-12 \mathrm{~Hz})$ and delta irregular activities $(0.5-4 \mathrm{~Hz})$ were measured from 7 animals. The peak frequencies of hippocampal theta and delta activities were not significantly altered in kindled animal relative to pre-kindling measurements $(p=0.723$ and $p=0.524$, paired test). 
addition, the animals exhibited hippocampal "physiological" activities similar to those we and others have previously observed in mice [15-20,22]. Specifically, CA3 recordings revealed rhythmic activities in the theta band $(5-12 \mathrm{~Hz})$ while the animals moved/explored local environments and irregular activities in the delta band $(0.5-4 \mathrm{~Hz})$ during immobility or sleep (Figure 2E-H). The peak frequencies of hippocampal theta and delta activities were not significantly changed in kindled animals $(n=7$ mice; Figure $2 \mathrm{H})$. As bilateral hippocampi are strongly interconnected via ventral and dorsal hippocampal fissures and the CA3 area is critical for the generation of hippocampal EEG rhythms and epileptiform activities [23], the above data provide electrographic evidence for accurately implanted CA3 electrodes.

The locations of implanted CA3 electrodes were histologically confirmed in 6 animals. The tracks of implanted electrodes were recognizable in brain sections that corresponded to the desired stereotaxic coordinates (Figure 1C). There was no evident loss of hippocampal neurons in the areas near the implanted electrodes or in adjacent brain sections (Figure 1D). Taken together these observations with the electrographic data above, we suggest that the screw-free and glue-based method is suitable for reliable implantations of bipolar stimulating/ recording electrodes in adult mice.

\section{Seizure activities induced by hippocampal kindling}

We used a standard kindling protocol in the present experiments $[24,25]$. The animals were considered fully kindled if they exhibited stage 5 behavioral seizures on 3 consecutive days. Of 25 animals tested, 21 animals were fully kindled between 6 and 26 stimulations (mean \pm SD $12.14 \pm 6.41$ ). Two of the remaining 4 animals failed to reach a fully kindled state due to contamination of implanted electrodes (see Discussion) and the other two died spontaneously with underlying causes presently unknown.

In addition to consistent expression of the stage 5 behavioral seizures, the kindled animals showed significant changes in hippocampal electrographic activities. First, the evoked field potentials were greatly enhanced in the kindled animals as compared to their own baselines (Figure 2A). Such enhancements were recognizable at all stimulation intensities examined (Figure 2B), suggesting an overall increase of hippocampal synaptic strength induced by the kindling process. Secondly, the afterdischarges (ADs) were greatly increased in amplitude and duration in the kindled animals (Figure 3A, C). Finally, interictal-like hippocampal spikes, similar to those previously described [26-29], were frequently observed in the kindled animals but were either absent or barely detectable in the same animals during baseline monitoring (Figure 2C-D).
The behavioral seizures and ADs observed from the kindled animals were sensitive to inhibition by midazolam, a fast acting benzodiazepine analogue recommended for the treatment of status epilepticus in clinical practice [30,31]. Midazolam was administered via intra-peritoneal injections at a dose of $2 \mathrm{mg} / \mathrm{kg}$, 15 minutes prior to the hippocampal stimulation. In the 6 kindled animals tested, ADs and behavioral seizures were significantly attenuated following midazolam injections compared to those observed following the saline injections (Figure 3A-C). Together, the above observations suggest that a robust epileptic process is established in the kindled mice.

\section{Discussion}

Using a modified version of the screw-free and gluebased method, we implanted bipolar electrodes in bilateral hippocampal CA3 areas. In our experiments, no animal had adverse health-related events, spontaneous seizures, or abnormal behavior in the absence of electrical stimulation. There was no local infection in the implanted area and no loss of implanted electrodes after repetitive stimulations and recordings for 4-6 months. The kindled animals exhibited consistent stage 5 behavioral seizures, robust ADs (Figure 3A-D), and frequent interictal-like spikes (Figure $2 \mathrm{C}-\mathrm{D}$ ). The $\mathrm{AD}$ and behavioral seizures were sensitive to suppression by midazolam (Figure 3A-C). We thus suggest that the modified glue method is reliable for intracranial electrode implantation, chronic brain stimulation and highquality EEG recordings in mice. Specific technical issues of the modified method are discussed below.

In our previous studies [14-20], we used a preconstructed electrode array with a plastic base and applied glue between the plastic base and skull surface. Because the glue bonds the plastic base strongly to the skull, the electrode array can be firmly mounted onto the skull without using anchoring screws. This method is convenient for implanting the electrode array with 23 monopolar electrodes, and allows stable intracranial EEG recordings in mouse models [15-20]. This method, however, is limited for implanting bipolar electrodes that are aimed to stimulate and/or record from spatially separated brain structures, since these electrodes are difficult to assemble in a compact array. In addition, due to the curvature of the skull, these electrodes need to be individually inserted into the brain for accurate positioning.

We thus used a modified approach in the present experiments. We first glued a plastic base onto the skull surface and drilled small holes through the plastic base and underneath skull. We then individually positioned bipolar electrodes in the brain and overlaid dental acrylic onto the plastic base covering the implanted electrodes. 

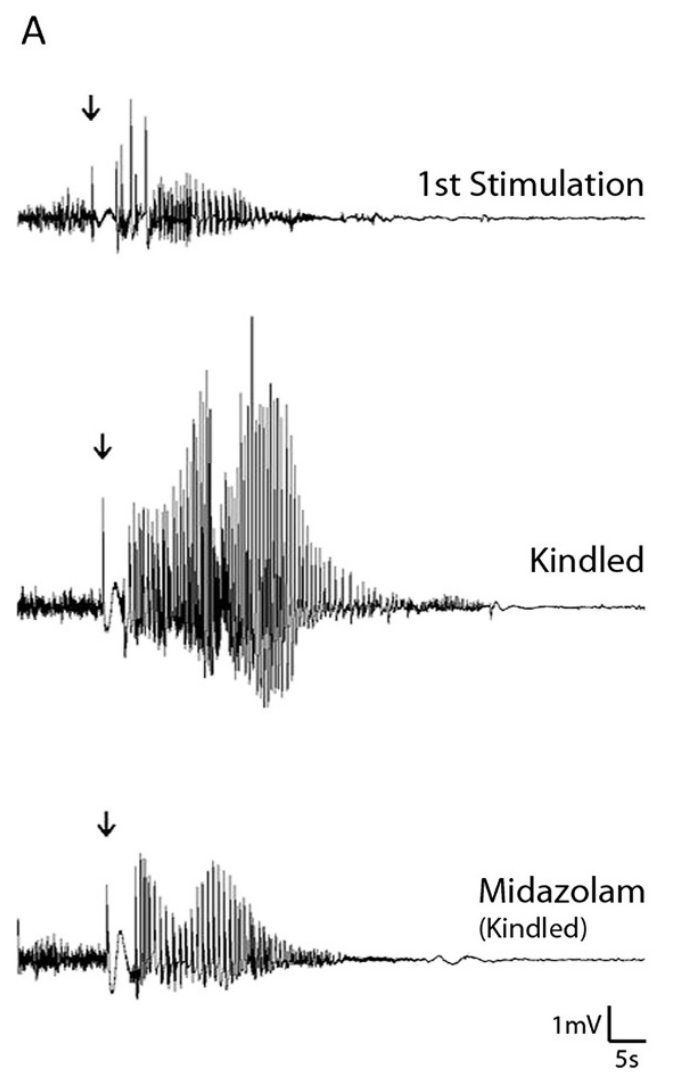

B

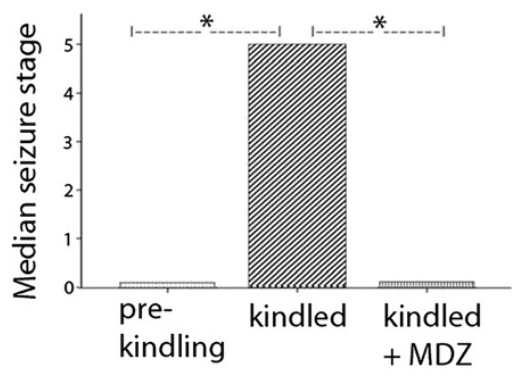

C

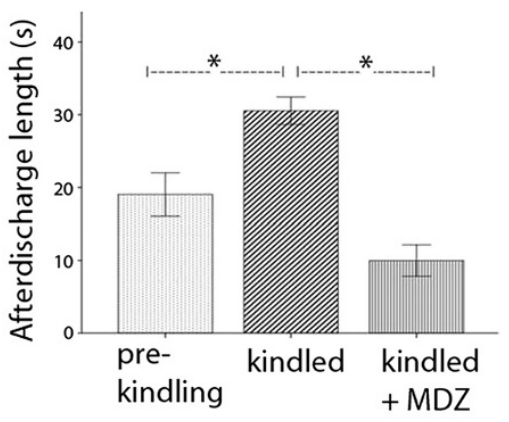

Figure 3 EEG discharges and motor seizures induced by hippocampal kindling. A, afterdischarges recorded from a mouse after the first stimulation (top) and fully kindled following an intra-peritoneal injection of saline (middle) or midazolam (MDZ; $2 \mathrm{mg} / \mathrm{kg}$, bottom). The afterdischarges were evoked by unilateral CA3 stimulation ( $60 \mathrm{~Hz}$ for 2 seconds, indicated arrows) and recorded from the contralateral CA3 area. B, seizure stages assessed from 6 animals. ${ }^{*}, p=0.008$ or $p=0.014$; Wilcoxon signed rank test. , afterdischarges measured from same 6 animals. *, $p=0.014$; paired t tests.

When hardened, the dental acrylic and the plastic base beneath the acrylic were strongly bonded; this secured the implanted electrodes (Figure 1A-B). This modified approach is successful because dental acrylic denatures the plastic base before hardening and then bonds to the plastic base after hardened. The implanted electrodes are secured onto the skull when the dental acrylic has hardened. Experience is needed, however, to promptly overlay the dental acrylic onto the plastic base, and to carefully cover the implanted electrode bases, before the acrylic hardens. We used a dental acrylic with hardening time of 6-9 minutes in the present experiments to have sufficient time to apply the dental acrylic.

In the present experiments, the pins used to connect electrodes were detached from standard IC sockets (Figure 1A). We chose these pins because the IC sockets are widely available from commercial sources at a low cost. In addition, these pins are relatively strong and offer good contacts when connected. Thus, although animals frequently push or press these pins against their cages or metal food racks, the implanted electrodes maintain integrity for chronic stimulation and/or recordings. Care should be taken, however, when detaching these pins from wire sockets, to preserve a gasket-like piece in the female end of individual pins.

We implanted bipolar electrodes in bilateral hippocampal CA3 areas in the present experiments. This experimental design was used to test the possibility of stimulating two brain regions, and/or to increase the probability of electrical kindling in individual animals in the event that one bipolar electrode fails. The latter case was encountered in our initial experiments, likely due to electrode contamination that occurred during and/or after implantation. Such a problem did not occur in our later experiments, by visual inspection of electrode tips under a microscope and measurements of electrode conductivity before implantation. Therefore, a careful examination of the constructed electrodes prior to surgical implantation may greatly increase the success rate of chronic brain stimulation or recording.

Several previous studies have used cyanoacrylate-type glue alone or together with dental acrylic to secure EEG recording as well as stimulating electrodes in mouse models [32-34]. However, the underlying procedures 
were not detailed. It is possible that the electrode implantation methods used in these previous studies and our present experiments may share some common features. By detailing the glue-based implantation method, our present study may support the previous studies and provide complementary technical aspects for further employment of this approach in mouse models.

\section{Conclusion}

We suggest that the glue-based and screw-free implantation method is reliable for chronic brain stimulation and high-quality EEG recordings in mice. The method may also be used, with some modifications, to implant other probes or infusion tubes in the mouse brain.

\section{Methods}

\section{Animals}

Male C57 black mice (initial ages of 6-8 months, Charles River Laboratory, Quebec, Canada) were used in the present experiments. These animals were housed in a vivarium that was maintained at $22^{\circ} \mathrm{C}$ with 12 -hour light and dark cycles. Food and water were available $a d$ libitum. Stimulations and recording experiments were done between 12-5 pm. All experimental procedures described below have been reviewed and approved by the Animal Care Committee of the University Health Network in accordance with the guidelines of the Canadian Council on Animal Care.

\section{Electrode construction}

All electrodes were made of polyamide-insulated stainless steel wires (outer diameter $200 \mu \mathrm{m}$, Plastics One, Ranoake, VA). Twisted bipolar wires were used for stimulation and recording, with their intracranial tips $\sim 100 \mu \mathrm{m}$ apart. The extracranial tips of the twisted wire assembly were soldered to the female ends of two connecting pins, with one wire bent into an L-shape to separate the connecting pins (Figure 1A). Care was taken to fully remove the insulation layer before soldering. A liquid solder (Soldering Liquid Flux, Certanium Alloys and Research Company, Cleveland OH, USA) or weak phosphoric acid was used to ensure good contact between the stainless steel wire and the connecting pin. A single wire was similarly soldered to a single pin for reference. The resistance of each constructed wire electrode was $\leq 1.0 \Omega$. After being soldered to the connecting pins, the bipolar wires were then cut to $\sim 3 \mathrm{~mm}$ in length to target the desired hippocampal CA3 area (see below). The single monopolar wire was cut to $\leq 0.5 \mathrm{~mm}$ for epidural position of the reference electrode. These electrodes were cleaned with $75 \%$ alcohol and stored in a sterilized glass vial until use. The connecting pins were detached from standard IC sockets (Samtec series SS socket strips, SS-132-G2, Electrosonic,
Toronto, Ontario, Canada). These pins are $7.5 \mathrm{~mm}$ long in total; the male end of the pin is $3.2 \mathrm{~mm}$ long. The outer diameter is $1.8 \mathrm{~mm}$ or $0.5 \mathrm{~mm}$ for female or male end of the pin respectively (Figure 1A). Measured after being dissected out from implanted animals $(n=3)$, the total weight of implanted electrode assembly, including electrode wires, plastic base and dental acrylic (see below), was 0.50-0.52 grams. As adult mice were used in the present experiments, the weight of implanted electrode assembly was $\leq 2 \%$ of animal body weight.

\section{Surgery for electrode implantation}

Surgical procedures were modified from those we previously described [14,21]. Briefly, the animal was anaesthetized with $2 \%$ isoflurane and then placed on a stereotaxic frame. After skin incision and exposure of the skull surface, the tip of a mini drill bit (see below) was aimed to bregma via a micromanipulator. After determining the bregma position, the drill bit was moved up but its X-Y position was unchanged, and a thin plastic base then was glued onto the skull surface. After the glue had cured, three small holes were then drilled through the plastic base and the skull according to the stereotaxic coordinates of the hippocampal CA3 area (bregma $-2.5 \mathrm{~mm}$, lateral $\pm 1.3 \mathrm{~mm}$, and a depth of $3.2 \mathrm{~mm}$ [35]). The reference electrode was positioned at bregma $1 \mathrm{~mm}$, lateral $2.0 \mathrm{~mm}$ and a depth of $0.7 \mathrm{~mm}$. The electrode depths were adjusted to accommodate for the thickness $(200 \mu \mathrm{m})$ of the plastic base.

The plastic base was cut from a curved part of plastic weighting boats (polystyrene antistatic weighting dishes, Fisher Scientific, cat\#08-732-115). The weighting boats were $140 \times 140 \mathrm{~mm}$ in length-width and $25 \mathrm{~mm}$ in depth, with thickness of $\sim 200 \mu \mathrm{m}$. The plastic base was soft and could be gently pressed to accommodate curvature of the skull, and thus bound tightly with the skull surface after being glued. We used a cyanoacrylate-type glue (Insta-cure+, cure time 5-15 seconds, made in U.S. A., cat\# BSI-106C; obtained from Canadian Hobbycraft, Concord, Ontario, Canada). A motorized drill (model FM3545, Foredom Electric, Bethel, CT, USA) and a mini drill bit (part 115603, Ball Mills Carbide, CircuitMedic, Haverhill, MA, USA) were used to drill small holes $(\leq 0.5$ $\mathrm{mm}$ ) through the skull. These holes were large enough for inserting the electrodes, but small enough to prevent dental acrylic leakage into the brain (see below).

Micromanipulators were also used to individually insert the bipolar electrodes into bilateral hippocampal CA3 areas. After positioning and holding these electrodes with the micromanipulators, dental acrylic was overlaid onto the plastic base such that the bases of the connecting pins were covered by the dental acrylic (Figure 1B). Care should be taken to apply acrylic so as not to interfere with electrode positions and to 
contaminate connecting pins. We used a dental acrylic with hardening time of 6-9 minutes (Jet Tooth Shade, Reference No. 1404; Lang Dental Mfg. Co., Inc., Wheeling, IL, USA) to carefully cement the implanted electrodes. After the dental acrylic had hardened, the electrodes were released from the micromanipulators. The incised skin was then glued to the dental acrylic (Figure 1A,1B), which prevents infection in the implanted area. The animals were released from the stereotaxic frame and allowed to recover for at least one week before further experimentation.

\section{Electrical stimulation}

Unilateral CA3 stimulation was conducted in all present experiments. Constant square-wave current pulses (duration of $0.5 \mathrm{~ms}$, intensities of $10-150 \mu \mathrm{A}$ ) were generated from a Grass stimulator and delivered through an isolation unit (model S88H, Grass Medical Instruments, Warwick RI, USA). To establish an input-output plot for evoked CA3 field potentials, a single stimulation was applied every 30 seconds at intensities of $10-150 \mu \mathrm{A}$ $(10 \mu \mathrm{A}$ increments; 5 consecutive responses at a given intensity). A standard kindling protocol [24,25] was used. Animals were considered fully kindled after consecutive stage 5 seizures on 3 consecutive days.

\section{Recordings and measurements}

All recordings were from the CA3 area contralateral to the CA3 stimulation site. Signals were recorded via a 2channel microelectrode AC amplifier (model 1800, A-M Systems, Carlsborg, WA, USA), with the input frequency band set in the range of $0.1-1000 \mathrm{~Hz}$, and the amplification gain at $1000 \times$. The signals were digitized at $5000 \mathrm{~Hz}$ (Digidata 1440A, Axon Instruments/Molecular Devices, Union City, CA, USA). Pclamp software (Version 9 or 10; Axon Instruments/Molecular Devices) was used for data acquisition, storage and analyses.

The amplitudes of evoked field potentials were measured from averaged traces (5 consecutive responses) at a given stimulation intensity. To measure hippocampal rhythmic activities associated with "active" and "inactive" behaviors, stable data segments of 5-10 seconds or 3060 seconds were selected while the animals were moving/exploring or immobile/asleep. Spectral plots were generated from these data segments and peak frequencies were measured from the spectral plots for individual animals. To detect interictal-like spikes, individual animals were recorded for up to four hours before kindling was initiated. After animals were fully kindled, interictal spikes were recorded for 4-6 hours after the most recent ADs. A spike was only counted if its amplitude was large ( $\geq 2$ times the amplitude of the background signal) and its waveform was similar to those previously described [26-29]. To minimize interference of movement-related artifacts, the rates of interictal spikes were measured in the periods (1-2 hours) while the animals were immobile/asleep. To measure AD durations, corresponding EEG data were treated with a $0.5 \mathrm{~Hz}$ high-pass (Bessel) filter to reduce slow drifts in EEG signals.

\section{Behavioral assessments}

Animal's behaviors were recorded with a high definition camera and analyzed by blinded experimenters. Behavioral seizures were scored using a modification of the Racine scale for the mouse [25]: stage 0, no response or behavioral arrest; stage 1, chewing or head-nodding; stage 2, chewing and head nodding; stage 3, single or bilateral forelimb clonus; stage 4 , bilateral forelimb clonus and rearing; stage 5, loss of righting reflex (falling).

\section{Brain histology}

Histological experiments were conducted as we previously described [15,17]. Briefly, the animals were anesthetized with pentobarbital $(70 \mathrm{mg} / \mathrm{kg}$, i.p.) and transcardiacally infused with saline and then with $10 \%$ phosphate-buffered formalin solution before decapitation. Cryostat coronal sections $30 \mu \mathrm{m}$ thick were obtained throughout the brain, stained with cresyl violet, and examined under a light microscope.

\section{Statistical analysis}

Statistical tests were performed with SPSS software (Version 20, SPSS Statistics, IBM). Data are presented as mean and standard error of mean $(\mathrm{M} \pm \mathrm{SE})$ throughout the text and figures except where indicated.

\section{Abbreviations}

AD: After-discharges; CA3: Cornu ammonis 3 area; EEG: Electroencephalography.

\section{Competing interests}

The authors of this study declare that they have no competing interests.

\section{Authors' contributions}

$\mathrm{MJ}$ and $\mathrm{ML}$ made equal contributions to this study. All authors were involved in experimental design, background/literature search and conceptualizations of this study. Specifically, MJ, ML and JG conducted experiments and data analysis. CW constructed electrodes and conducted surgeries for electrode implantation. MJ, ML and LZ assembled manuscript. $M B$ edited the manuscript. All authors have read and approved the final manuscript.

\section{Authors' information}

MJ-PhD student at the Department of Pharmacology, University of Toronto. ML-MSc, Research fellow, Toronto Western Research Institute. JG-Undergraduate student at the University of Toronto.

CW-Research associate at the Toronto Western Research Institute. MB-Professor at the Department of Pharmacology, University of Toronto. LZ-Research Scientist at the Toronto Western Research Institute.

\section{Acknowledgements}

This study was funded by research grants from the Natural Science and Engineering Research Council (NSERC) of Canada to LZ and from the Ontario Brain Institute $(\mathrm{OBI})$ to $\mathrm{LZ}$ and $\mathrm{BM} . \mathrm{MJ}, \mathrm{ML}, \mathrm{JG}$ and $\mathrm{CW}$ were supported fully or partly by the NSERC and OBI grants. Funding sources were not involved 
in experimental design, data collection and interpretation, analysis, writing of the report, or the decision to submit for publication.

\section{Author details}

${ }^{1}$ Toronto Western Research Institute, University Health Network, Toronto, Ontario, Canada. ${ }^{2}$ Department of Pharmacology, Toronto, Ontario, Canada. ${ }^{3}$ Department of Medicine (Division of Neurology), University of Toronto, Toronto, Ontario, Canada. ${ }^{4}$ University of Toronto Epilepsy Research Program, Toronto, Ontario, Canada.

Received: 22 March 2013 Accepted: 2 August 2013

Published: 6 August 2013

\section{References}

1. McIntyre DC, Poulter MO: Kindling and the mirror focus. Inter Rev Neurobiol 2001, 45:387-406.

2. Morimoto K, Fahnestock M, Racine RJ: Kindling and status epilepticus models of epilepsy: rewiring the brain. Prog Neurobiol 2004, 73:1-60.

3. Bialer M, White HS: Key factors in the discovery and development of new antiepileptic drugs. Nat Rev Drug Discovery 2010, 9:68-82.

4. Löscher W: Critical review of current animal models of seizures and epilepsy used in the discovery and development of new antiepileptic drugs. Seizure 2011, 20:359-368.

5. Bragin $\mathrm{A}$, Wilson $\mathrm{CL}$, Engel J Jr: Increased afterdischarge threshold during kindling in epileptic rats. Exp Brain Res 2002, 144:30-37.

6. Lonsdale D, Nylen K, Burnham WM: The anticonvulsant effects of progesterone and its metabolites on amygdala-kindled seizures in male rats. Brain Res 2006, 1101:110-116.

7. Gavrilovici C, D'Alphonso S, Dann M, Poulter MO: Kindling-induced alterations in GABAA receptor-mediated inhibition and neurosteroid activity in the rat piriform cortex. Eur J Neurosci 2006, 24:1373-1384.

8. Hargus NJ, Merrick EC, Nigam A, Kalmar CL, Baheti AR, Bertram EH, Patel MK: Temporal lobe epilepsy induces intrinsic alterations in $\mathrm{Na}$ channel gating in layer II medial entorhinal cortex neurons. Neurobiol Dis 2011, 41:361-376.

9. Epps SA, Kroshona DT, Lin SJ, Kahn AB, Javors MA, Boss-Williams KA, Weiss $J M$, Weishenker D: Seizure susceptibility and epileptogenesis in a rat model of epilepsy and depression co-morbidity. Neuropsychopharmacol 2012, 37:1-8.

10. Frye $C A$, Rhodes ME, Walf A, Harvey J: Progesterone reduces pentylenetetrazol-induced ictal activity of wild-type mice but not those deficient in type I 5a-reductase. Epilepsia 2002, 43(Suppl. 5):14-17.

11. Gangisetty O, Reddy DS: Neurosteroid withdrawal regulates $G A B A_{A}$ receptor a4-subunit expression and seizure susceptibility by activation of progesterone receptor-independent early growth response factor-3 pathway. Neurosci 2010, 170:865-880

12. Manno I, Macchi F, Caleo M, Bozzi Y: Environmental enrichment reduces spontaneous seizures in the Q54 transgenic mouse model of temporal lobe epilepsy. Epilepsia 2011, 52(9):e113-e117.

13. Reddy DS, Mohan A: Development and persistence of limbic epileptogenesis are impaired in mice lacking progesterone receptors. J Neurosci 2011, 31(2):650-658.

14. Wu CP, Wais M, Sheppy E, Del Campo M, Zhang L: A glue-based, screwfree method for implantation of intra-cranial electrodes in young mice. J Neurosci Meth 2008, 171:126-131.

15. Wais M, Wu CP, Sheppy E, Del Campo M, Zhang L: Repeated hypoxic episodes induce seizures and alter hippocampal network activities in mice. Neurosci 2009, 161:599-613.

16. D'Cruz JA, Wu CP, Zahid T, Hayek J, Zhang L, Eubanks JH: Alterations of cortical and hippocampal EEG activity in MeCP2-deficient mice. Neurobiol Dis 2010, 38:8-16

17. El-Hayek YH, Wu CP, Chen R, Al-Sharif AR, Huang S, Patel N, Du C, Ruff CA, Fehlings MG, Carlen PL, Zhang L: Acute post-ischemic seizures are associated with increased mortality and brain damage in adult Mice. Cereb Cort 2011, 21:2863-2875.

18. Wither RG, Lang M, Brotchie JM, Wu CP, Zhang L, Eubanks JH: Regional MeCP2 expression levels in the female MeCP2-deficient mouse brain correlate with specific behavioral impairments. Exp Neurol 2013, 239:49-59.

19. Lang $M$, Wither $R G$, Brotchie JM, Wu C, Zhang L, Eubanks JH: Selective preservation of $\mathrm{MeCP} 2$ in catecholaminergic cells is sufficient to improve the behavioral phenotype of male and female Mecp2-deficient mice. Hum Mol Genet 2013, 22(2):358-371.

20. El-Hayek YH, Wu CP, Ye H, Wang J, Carlen PL, Zhang L: Hippocampal excitability is increased in aged mice. Exp Neurol 2013. doi:10.1016/j. expneurol.2013.03.012. in press.

21. Wu CP, Wais M, Zahid T, Wan Q, Zhang L: An improved screw-free method for electrode implantation and intra-cranial electroencephalographic recordings in mice. Behav Res Meth 2009, 41:736-741.

22. Buzsáki G, Buhl DL, Harris KD, Csicsvari J, Czéh B, Morozov A: Hippocampal network patterns of activity in the mouse. Neurosci 2003, 116(1):201-211

23. Witter MP: Intrinsic and extrinsic wiring of CA3: indications for connectional heterogeneity. Learn Mem 2007, 14(11):705-713.

24. Albright PS, Burnham WM: Development of a new pharmacological seizure model: effects of anticonvulsants on cortical- and amygdalakindled seizures in the rat. Epilepsia 1980, 21:681-689.

25. Reddy DS, Rogawski MA: Ganaxolone suppression of behavioral and electrographic seizures in the mouse amygdala kindling model. Epilepsy Res 2010, 89(2-3):254-260.

26. Kairiss EW, Racine RJ, Smith GK: The development of the interictal spike during kindling in the rat. Brain Res 1984, 322:101-110.

27. Leung LW: Hippocampal interictal spikes induced by kindling: relations to behavior and EEG. Behav Brain Res 1988, 31(1):75-84.

28. Leung LW: Spontaneous hippocampal interictal spikes following local kindling: time-course of change and relation to behavioral seizures. Brain Res 1990, 513(2):308-314

29. Timofeeva OA, Peterson GM: Bilateral hippocampal interictal spiking and kindling expression in rabbits. Epilepsy Res 1996, 25(3):149-159.

30. Meierkord H, Boon P, Engelsen B, Göcke K, Shorvon S, Tinuper P, Holtkamp M: EFNS guideline on the management of status epilepticus in adults. Eur J Neurol 2010, 17(3):348-355.

31. Shorvon S: The treatment of status epilepticus. Curr Opin Neurol 2011, 24:165-170.

32. Siegel SJ, Connolly P, Liang L, Lenox RH, Gur RE, Bilker WB, Kanes SJ, Turetsky Bl: Effects of strain, novelty, and NMDA blockade on auditoryevoked potentials in mice. Neuropsychopharmacol 2003, 28:675-682.

33. Featherstone RE, Liang $Y$, Saunders JA, Tatard-Leitman VM, Ehrlichman RS, Siegel SJ: Subchronic ketamine treatment leads to permanent changes in EEG, cognition and the astrocytic glutamate transporter EAAT2 in mice. Neurobiol Dis 2012, 47(3):338-346.

34. Saunders JA, Tatard-Leitman VM, Suh J, Billingslea EN, Roberts TP, Siegel SJ: Knockout of NMDA receptors in parvalbumin interneurons recreates autism-like phenotypes. Autism Res 2013. doi:10.1002/aur.1264 [Epub ahead of print].

35. Franklin KBJ, Paxinos G: The mouse brain in stereotaxic coordinates. San Diego (CA): Academic Press; 1997.

doi:10.1186/1471-2202-14-82

Cite this article as: Jeffrey et al.: A reliable method for intracrania electrode implantation and chronic electrical stimulation in the mouse brain. BMC Neuroscience 2013 14:82

\section{Submit your next manuscript to BioMed Central and take full advantage of:}

- Convenient online submission

- Thorough peer review

- No space constraints or color figure charges

- Immediate publication on acceptance

- Inclusion in PubMed, CAS, Scopus and Google Scholar

- Research which is freely available for redistribution 